\title{
The Effect and Safety of Steroid Injection in Lumbar Spinal Stenosis: With or Without Local Anesthetics
}

\author{
Sung Hyuk Song, MD', Gi Hyeong Ryu, MD², Jin Woo Park, MD, PhD ${ }^{1}$, Ho Jun Lee, MD, PhD', \\ Ki Yeun Nam, $\mathrm{MD}^{1}$, Hyojun Kim, $\mathrm{MD}^{1}$, Seung Yeon Kim, $\mathrm{MD}^{1}$, Bum Sun Kwon, MD, $\mathrm{PhD}^{1}$ \\ ${ }^{1}$ Department of Physical Medicine and Rehabilitation, Dongguk University College of Medicine, Goyang; \\ ${ }^{2}$ Doctor Ryu's Rehabilitation Clinic, Gyeongju, Korea
}

Objective To compare the long-term effect and safety of an epidural steroid injection in spinal stenosis patients, with or without local anesthetics.

Methods Twenty-nine patients diagnosed with spinal stenosis were included and randomly divided into two groups. Translaminar epidural and selective nerve root spinal injection procedures were performed using steroids mixed with local anesthetics or normal saline. The effects of spinal injection procedures were measured with visual analogue scale (VAS) and functional rate index (FRI). These measurements were performed before injection, at 1 month after injection and at 3 months after injection. The occurrence of side effects was investigated each time.

Results The VAS and FRI scores were significantly reduced in both the local anesthetics group and normal saline group at 1 and 3 months after the injection. However, there was no significant difference in VAS and FRI score reduction between the two groups each time. Side effects are not noted in both groups.

Conclusion The spinal injection procedures using steroids mixed either with local anesthetics or normal saline have an effect in reducing pain and improving functional activities. However, there was no significant difference between the two groups in relation to side effects and the long-term effects of pain and function.

Keywords Epidural injections, Spinal stenosis, Steroids, Anesthetics

Received May 21, 2015; Accepted July 17, 2015

Corresponding author: Bum Sun Kwon

Department of Physical Medicine and Rehabilitation, Dongguk University College of Medicine, 27 Dongguk-ro, Ilsandong-gu, Goyang 10326, Korea

Tel: +82-31-961-7460, Fax: +82-31-961-7488, E-mail: bskwon@dumc.or.kr

(c) This is an open-access article distributed under the terms of the Creative Commons Attribution Non-Commercial License (http://creativecommons. org/licenses/by-nc/4.0) which permits unrestricted noncommercial use, distribution, and reproduction in any medium, provided the original work is properly cited.

Copyright $\odot 2016$ by Korean Academy of Rehabilitation Medicine

\section{INTRODUCTION}

Spinal stenosis is a degenerative disease of the spine and can be classified as either central or lateral stenosis. A feature of central stenosis is a narrowing of the spinal canal that surrounds the dural sac. On the other hand, lateral stenosis patients exhibit narrowing near the spinal nerve or intervertebral foramen. There are many instances where these two types of spinal stenosis are combined. Spinal stenosis can result in the compression of nerves 
or vessels, which may evoke symptoms. Patients' symptoms and physical examinations are mandatory for the diagnosis of spinal stenosis, and additional examinations may be required such as radiography, electrodiagnostic examination, myelography, and computed tomography and magnetic resonance imaging (MRI) [1]. Conservative therapy can relieve the pain induced by spinal stenosis, but in some other cases, conservative therapy is not effective and alleviation of pain is achieved through surgical therapy [2].

Spinal stenosis is an important cause of lower back pain in the elderly and pain is frequently an important indicator for the need for surgical treatment. However, the ideal surgical treatment time is not certain and symptoms can still continue even after treatment. Moreover, surgical treatment can cause scar tissue formation that can result in restenosis of the spinal canal. In the elderly, the burden of anesthesia and possible post-operative complications should be considered before surgery because they may have had many other medical problems [3]. For this reason, translaminar epidural steroid injections (TLESIs) and selective nerve root injections (SNRIs) are good alternative therapies that have produced relatively effective results as reported by many researchers. These spinal injections have been reported to show improvements in $60 \%-75 \%$ of patients [4-7].

Local anesthetics are traditionally used in spinal steroid injection procedures, with $2 \%$ lidocaine hydrochloride being the most commonly used medication. However, it can induce some side effects such as severe hypotension, which is caused blocking of the sympathetic nerve and motor nerve, as well as ataxic movement, lower limb paralysis and respiratory inadequacy. Such adverse reactions due to lidocaine use have been reported to occur in about $1 \%-3 \%$ of cases $[8,9]$. In order to prevent such side effects, some have recommended the use of steroids mixed with saline for the procedure instead of steroids mixed with local anesthetics. However, there is no standardized protocol for whether or not the use of local anesthetics should be avoided in spinal injection procedures [5]. The purpose of this study is to compare the safety and the long-term effects of spinal injection procedures in patients with spinal stenosis using steroids with or without local anesthetics.

\section{MATERIALS AND METHODS}

\section{Subjects}

Fifty-three patients with a history of intermittent claudication and lower limb radicular pain or paresthesia were screened from October 2012 to January 2014. Patients diagnosed with spinal stenosis underwent MRI and electrodiagnostic examinations. Included patients had an anterior-posterior (AP) diameter of the spinal canal of less than $12 \mathrm{~mm}$ confirmed through sagittal imaging, and an AP foraminal diameter of less than $3 \mathrm{~mm}$ confirmed through parasagittal imaging, both by MRI $[1,10]$, and were found to have abnormal somatosensory evoked potentials (SEPs). SEPs were obtained at both of the saphenous nerves, the superficial peroneal nerves and sural nerves. For each nerve type, SEPs were obtained twice and the average of two latencies of the symptomatic side was compared with contralateral side. A latency delay of over 3 ms was defined as abnormal [11-13]. The electrodiagnostic study was performed using a certified machine (Medelec Synergy; Oxford Instruments, Surrey, UK). Among the patients presenting typical symptoms of spinal stenosis that were confirmed by MRI and SEPs, we excluded patients who had a lower limb vascular disorder, a psychological problem, another musculoskeletal disorder or symptoms of a neurogenic bladder or bowel. After exclusion of these groups, 29 patients (14 males, 15 females) participated in this study and were randomly divided into two groups using a one-to-one randomization method. One group received steroids mixed with local anesthetics (lidocaine group) and the other group used steroids mixed with normal saline (saline group). The use of lidocaine was unnoticed in either group (Fig. 1). All study designs were approved by the Institutional Review Board of Dongguk University College of Medicine.

\section{Intervention}

Because all patients had both central and lateral spinal stenosis, both TLESIs and SNRIs were carried out on every patient in both groups. In the lidocaine group, $40 \mathrm{mg}$ triamcinolone mixed with $10 \mathrm{~mL} 0.5 \%$ lidocaine was used in TLESIs under the guide of fluoroscopy. SNRIs with 10 mg dexamethasone mixed with $2 \mathrm{~mL} 0.5 \%$ lidocaine were injected at the level of dermatome with abnormal SEPs. In the saline group, an equal volume of saline was used instead of lidocaine for both TLESIs and SNRIs [6]. 


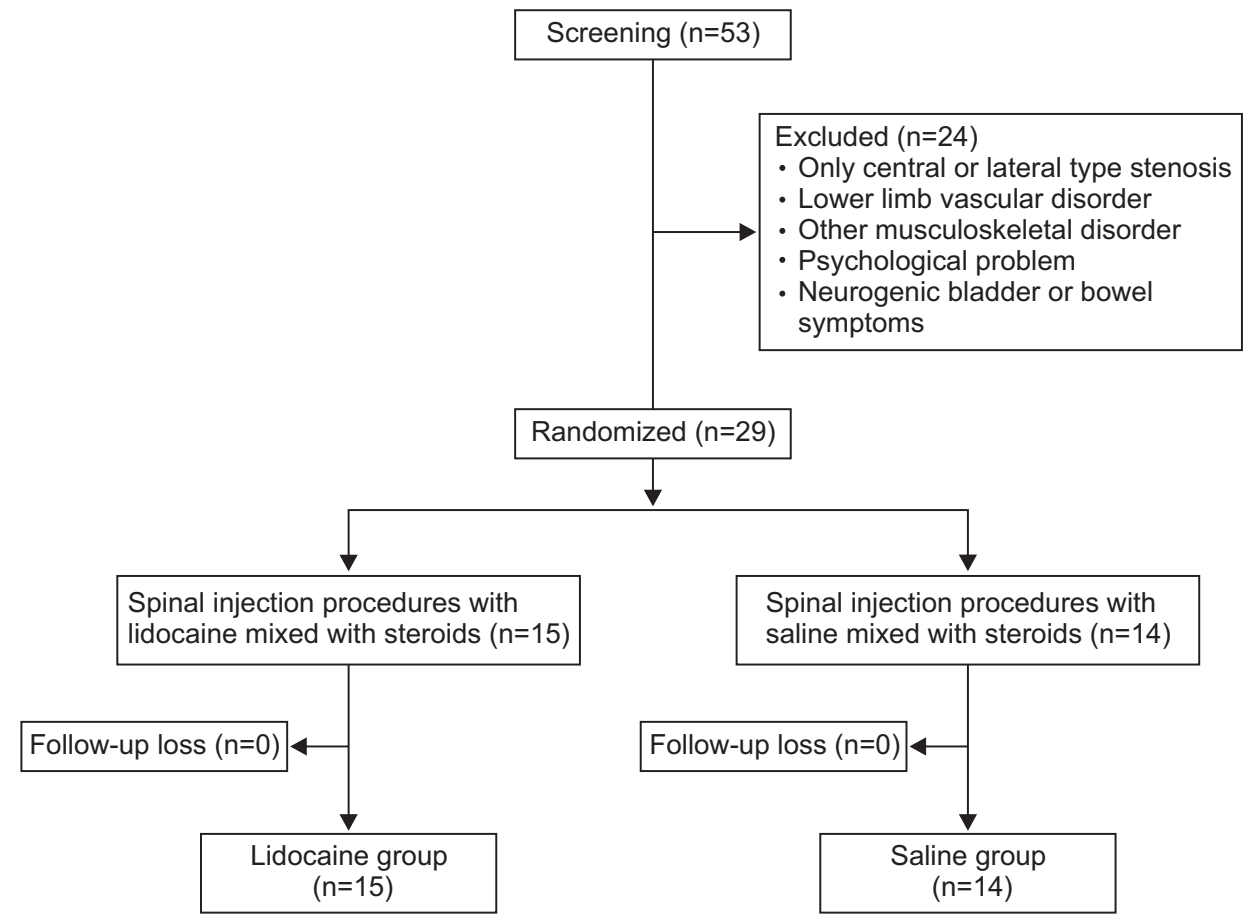

Fig. 1. Flow chart of study design. Fifty-three patients were screened for this study. Twenty-nine patients participated, and were divided randomly into two groups. There was no follow-up loss in both groups.
Before each procedure, local anesthetics were applied to the injection site. TLESI was done under fluoroscopy using a 22-gauge spinal needle and a posterior median approach was used to access the epidural space. Epidural space was identified with a loss of resistance technique and a contrast injection was carried out to confirm the location of the spinal needle tip. A steroid injection with lidocaine or saline was then carried out [14]. SNRI was also done under fluoroscopy using a 22-gauge spinal needle. Patients were lying prone at an angle of $45^{\circ}$ for identification of the foramen of the target level. The SNRI was then performed using the preganglionic approach as confirmed by contrast injection [15]. During the spinal injection procedures, each patient's respiratory rate, pulse rate, and blood pressure were monitored. After the procedure was completed, all patients took a rest in the supine position for 30 minutes, and were checked for vital signs and any adverse events. TLESI and SNRI were done once a week for three times. Every patient underwent injection for three times in order to compare both the positive and adverse effects of each procedure in the same condition.

\section{Outcome measures}

Pain intensity was measured with the visual analogue scale (VAS) and functional status was measured with the functional rate index (FRI). Each of these was checked before injection, at 1 month after injection, and at 3 months after injection. VAS is a unidimensional method of checking pain intensity and is measured using a range from 0 to 10 points. No pain is 0 points and the worst imaginable pain corresponds to 10 points, with patients choosing the current intensity of pain [16]. FRI is composed of 10 domains that measure functional status on a scale from 0 to 4 points. Full ability to function receives a score of 0 points while inability to perform functions corresponds to 4 points [17-19]. The number of injection failures with a less than $50 \%$ improvement in the VAS score was investigated after the procedures [7]. Additionally, we investigated whether side effects occurred immediately after injection, and at follow-up at 1 and 3 months later.

\section{Statistical analysis}

Statistical analyses were performed with SPSS-K ver. 20.0 (IBM SPSS, Armonk, NY, USA). In order to compare the demographics between the two groups, a chi-square for trend test was used for age and the number of abnormal root levels in the SEPs domain, and a chi-square test was used for the sex rate domain. Other domains such as the number of abnormal root levels for physical examinations, initial VAS score and initial FRI score were com- 
pared using a Mann-Whitney test.

A Friedman test was used for analyzing the effects of spinal injection procedures using the VAS and FRI scores for each group. Fisher exact test was used to compare injection failure between the two groups. A Mann-Whitney test was also used to compare effects between the lidocaine and saline groups. A p-value of $<0.05$ was considered statistically significant and a confidence interval of $95 \%$ was used.

\section{RESULTS}

The characteristics of patients are described including age, sex, number of abnormal root levels in physical examinations, number of abnormal root levels in SEPs, initial VAS score, and initial FRI score. There was no significant difference in the demographics of the two groups (Table 1).

The VAS scores of the lidocaine and saline groups at baseline were $4.5 \pm 1.7$ and $5.1 \pm 1.2$, respectively, and improved to $1.7 \pm 1.2$ and $2.9 \pm 1.5$ at 1 month and $3.0 \pm 1.8$ and $3.8 \pm 1.7$ at 3 months. Both groups showed a statistically significant reduction at 1 month and 3 months as compared with initial VAS scores $(\mathrm{p}<0.05)$. However, a comparison of the VAS score reduction between the two groups showed no significant difference at 1 month after injection ( $\mathrm{p}=0.696)$ and 3 months after injection $(\mathrm{p}=0.891)$ (Fig. 2). No complaints of pain or soreness at the injection sites were reported in both groups.

The FRI scores of the lidocaine and saline groups

Table 1. Summarizes demographic data of lidocaine and saline groups

\begin{tabular}{|lccc}
\hline & Lidocaine group & Saline group & p-value \\
\hline Age (yr) & $58.3 \pm 15.1$ & $61.7 \pm 14.8$ & 0.188 \\
\hline Sex (male:female) & $6: 9$ & $8: 6$ & 0.356 \\
No. of abnormal root levels in physical examinations & $2.8 \pm 1.2$ & $3.1 \pm 1.1$ & 0.075 \\
No. of abnormal root levels in SEPs & $1.7 \pm 0.5$ & $1.8 \pm 0.6$ & 0.783 \\
\hline Initial VAS score & $4.5 \pm 1.7$ & $5.1 \pm 1.2$ & 0.258 \\
\hline Initial FRI score & $18.9 \pm 7.1$ & $22.4 \pm 5.0$ & 0.155 \\
\hline
\end{tabular}

Values are presented as mean \pm standard deviation or number.

SEPs, somatosensory evoked potentials; VAS, visual analogue scale; FRI, functional rate index.

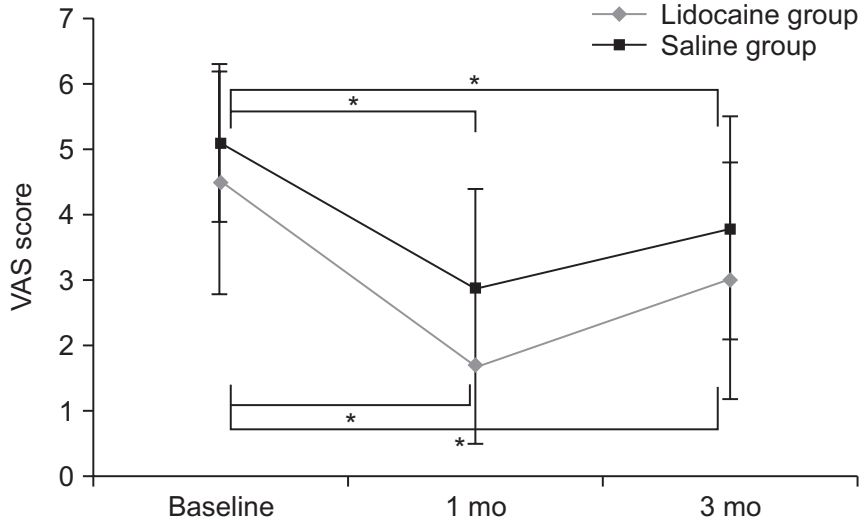

Fig. 2. Trend of visual analogue scale (VAS) score in lidocaine and saline groups. Trend of VAS score in the two groups before injection, and at 1 and 3 months after injection. Compared with the initial VAS score, both groups showed a significant reduction in VAS score at 1 month and 3 months after injection $\left({ }^{*} \mathrm{p}<0.05\right)$. A significant difference in VAS reduction was not noted between the two groups.

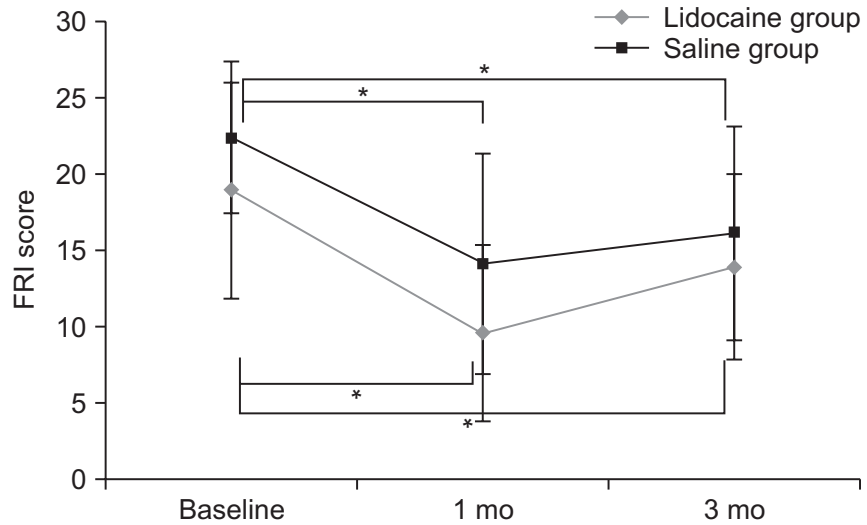

Fig. 3. Trend of functional rate index (FRI) score in lidocaine and saline groups. Trend of FRI score in two groups before injection, and at 1 and 3 months after injection. Compared with the initial FRI score, both groups showed a significant reduction in FRI score at 1 month and 3 months after injection $\left({ }^{*} \mathrm{p}<0.05\right)$. There was no significant difference in FRI score reduction between the two groups. 
at baseline were $18.9 \pm 7.1$ and $22.4 \pm 5.0$, respectively, and improved to $9.6 \pm 5.8$ and $14.1 \pm 7.2$ at 1 month and $13.9 \pm 6.1$ and $16.1 \pm 7.0$ at 3 months. Both groups showed the significant decreases in FRI scores at 1 month and 3 months after injection $(\mathrm{p}<0.05)$. However, there was no significant difference in FRI score reduction between the two groups at 1 month $(\mathrm{p}=0.983)$ and 3 months after injection ( $p=0.743)$ (Fig. 3$)$.

The number of injection failures, with a less than $50 \%$ improvement in the VAS score, was 3 for the lidocaine group and 4 for the saline group at 1 month after injection. At 3 months after injection, the number of injection failures was 8 in both groups. Thus, injection failure rate was $20.0 \%$ and $28.6 \%$ at 1 month, and $53.3 \%$ and $57.1 \%$ at 3 months, respectively. There was no significant difference in the injection failure rate of the two groups ( $\mathrm{p}=0.682$ at 1 month, $\mathrm{p}=0.837$ at 3 months).

We investigated the occurrence of side effects in all participating patients. It was found that the patients in both groups did not have any side effects immediately after injection, and 1 and 3 months later.

\section{DISCUSSION}

For diagnosis of spinal stenosis, all of the following are important: medical history, physical examinations, imaging studies, and electrodiagnostic studies. Medical history taking and physical examinations primarily can make differential diagnosis of other diseases. Imaging studies are essential, with MRI now being the standard examination tool for spinal stenosis [20]. The inclusion criteria of this study were an AP diameter of the spinal canal of less than $12 \mathrm{~mm}$, confirmed by sagittal imaging, and an AP foraminal diameter of less than $3 \mathrm{~mm}$, confirmed by parasagittal imaging. Another method for diagnosing spinal stenosis using MRI images also exists that involves measuring the cross-sectional area of the dural sac. We chose to use the AP diameter of the spinal canal or intervertebral foramen as diagnostic criteria because use of the cross-sectional area at the most narrowing part of the spinal canal may induce inter-rater difference at measurement. The patients enrolled in this study were all diagnosed with a combination of both central and lateral spinal stenosis. Electrodiagnostic studies can also be used for diagnosis of spinal stenosis, with bilateral and multi-segmental abnormalities being commonly found. These studies can help to identify the root level that requires treatment $[1,20]$. In our study, SNRIs were performed at abnormal SEP levels. Because abnormal needle electromyography levels were all included in abnormal SEP levels, we chose the inclusion criteria as only abnormal SEPs. Because misdiagnosis of spinal stenosis could affect the spinal injection procedures, MRI and SEP studies of abnormalities were use to make certain the diagnosis of spinal stenosis and the effect of the TLESIs and SNRIs.

The factors that determine the extent and intensity of the pain in spinal stenosis patients are inflammation and combined pressure or mechanical stimulation at the nerve roots. Howe et al. [21] reported that progression of the inflammatory process induces nerve root sensitization which results in continuing pain signal generation only at mild stimulation. Therefore, inflammation is thought to play an important role in the occurrence of back and lower limb pain in spinal stenosis patients, and the steroids' role in treatment is not only to inhibit the synthesis or release of proinflammatory substances, but also to reduce the production of arachidonic acid and its metabolites (prostaglandin and leukotriene), which consequently inhibits the inflammatory process and results in the reduction of pain in spinal stenosis patients. The results of this study show improvements in the VAS and FRI scores at 1 month and 3 months after injection, which has relevance of steroids' effect. When it comes to the total number of epidural steroid injections, McLain et al. [5] suggested the use of repeated injections for cases where the patient gets partial improvement from the procedure. On the other hand, if there has been a full relief of symptoms he insisted that the patient should not receive further injections. Injections were carried out three times for this study because the first and second procedures could not reach full relief of symptoms. Patients who did not receive injections three times were excluded.

A previous study has reported that a more favorable epidural steroid injection response was associated with relative youth and being female [22]. In this study, among the 7 subjects who showed injection failure at 1 month, 6 patients were male and 5 patients were older than the mean age. Several studies have reported that the effect of epidural steroids was maintained for weeks to years [5], and in this study, the VAS score tended to increase 3 months after injection, which resulted in a more than 
$50 \%$ injection failure rate in each group. Such an occurrence of injection failure is maybe due not only to increased pressure and mechanical stimulation in old age-as the severity of spinal stenosis gets worse with degenerative change-but also a limitation of efficacy duration of the steroids itself.

The expected pain generation mechanism at the time of nerve damage is an occurrence of inappropriate signals at the slow $\mathrm{Na}+$ channels [23]. The role of lidocaine in pain reduction is as an inhibitor of abnormal highfrequency neuronal discharges $[24,25]$. There is also another report that lidocaine's ability to reduce muscle spasms consequently helps to reduce pain [5]. Due to these effects, patients in the lidocaine group tended to have reduced pain before discharge. As such, patients felt satisfied after their spinal injection procedures and could increase their intimacy with the medical team. However, lidocaine has a short half-life meaning that its effect on pain is temporary. The use of lidocaine also causes side effects such as nausea, allergic reaction, hypotension, headache, lower limb paralysis, and ataxia. Moreover, if an excessive dosage is used, systemic reactions including vasovagal reaction, loss of consciousness, convulsions and respiratory depression can also occur $[8,9]$. In our study, there was no significant difference in long-term effects between the lidocaine and saline groups. We therefore recommend a saline injection mixed with steroids for patients who may have problems with the side effects of lidocaine. Such patients include those who have had abnormal spinal anatomy after undergoing spine surgery, underlying disease of asthma or food allergy, large body habitus, degenerative change of disc height loss, or facet hypertrophy and cardiopulmonary problems.

There are some limitations in this study. At first, as a noted above, the purpose of this study was to compare the long-term effect and safety of the injections between lidocaine and saline groups. We therefore did not check the numerical VAS score shortly after injection. Instead, before the patients were discharged, we questioned them about the pain and found out that the lidocaine group had a tendency toward earlier pain reduction. Secondly, maybe the most important limitation is that the number of patients who participated in this study is not adequate. Further studies with a larger number of participants will be needed.

In conclusion, spinal injections using steroids mixed with local anesthetics or normal saline both have an effect in reducing pain and improving functional activities. Due to the rapid effect in pain reduction, using local anesthetics can have the advantage in increasing affinity and rapport with patients. However, there was no significant difference in the long-term effects between both groups. Therefore, in consideration of the potential side effects of local anesthetics, we would prefer to use normal saline mixed with steroids for spinal injection procedures.

\section{CONFLICT OF INTEREST}

No potential conflict of interest relevant to this article was reported.

\section{REFERENCES}

1. Fritz JM, Delitto A, Welch WC, Erhard RE. Lumbar spinal stenosis: a review of current concepts in evaluation, management, and outcome measurements. Arch Phys Med Rehabil 1998;79:700-8.

2. Bush K, Hillier S. A controlled study of caudal epidural injections of triamcinolone plus procaine for the management of intractable sciatica. Spine (Phila Pa 1976) 1991;16:572-5.

3. Deyo RA, Cherkin DC, Loeser JD, Bigos SJ, Ciol MA. Morbidity and mortality in association with operations on the lumbar spine: the influence of age, diagnosis, and procedure. J Bone Joint Surg Am 1992;74:536-43.

4. Cirak B, Alptekin M, Palaoglu S, Ozcan OE, Ozgen T. Surgical therapy for lumbar spinal stenosis: evaluation of 300 cases. Neurosurg Rev 2001;24:80-2.

5. McLain RF, Kapural L, Mekhail NA. Epidural steroid therapy for back and leg pain: mechanisms of action and efficacy. Spine J 2005;5:191-201.

6. Nam KY, Ryu GH, Choi JM, Choi JH, Kwuan BS, Park JW. Efficacy of translaminar epidural steroid injection versus combined translaminar epidural steroid injection with selective nerve root injection in spinal stenosis. J Korean Acad Rehabil Med 2007;31:7-13.

7. Manchikanti L, Cash KA, McManus CD, Pampati V, Benyamin RM. Preliminary results of a randomized, double-blind, controlled trial of fluoroscopic lumbar interlaminar epidural injections in managing chronic 
lumbar discogenic pain without disc herniation or radiculitis. Pain Physician 2010;13:E279-92.

8. Botwin KP, Gruber RD, Bouchlas CG, Torres-Ramos FM, Freeman TL, Slaten WK. Complications of fluoroscopically guided transforaminal lumbar epidural injections. Arch Phys Med Rehabil 2000;81:1045-50.

9. Benedetti EM, Siriwetchadarak R, Stanec J, Rosenquist RW. Epidural steroid injections: complications and management. Tech Reg Anesth Pain Manag 2009;13:236-50.

10. Ericksen S. Lumbar spinal stenosis: imaging and non-operative management. Semin Spine Surg 2013; 25:234-45.

11. Zileli B, Ertekin C, Zileli M, Yunten N. Diagnostic value of electrical stimulation of lumbosacral roots in lumbar spinal stenosis. Acta Neurol Scand 2002;105:221-7.

12. Storm SA, Kraft GH. The clinical use of dermatomal somatosensory evoked potentials in lumbosacral spinal stenosis. Phys Med Rehabil Clin N Am 2004;15:107-15.

13. Snowden ML, Haselkorn JK, Kraft GH, Bronstein AD, Bigos SJ, Slimp JC, et al. Dermatomal somatosensory evoked potentials in the diagnosis of lumbosacral spinal stenosis: comparison with imaging studies. Muscle Nerve 1992;15:1036-44.

14. Manchikanti L, Cash KA, McManus CD, Damron KS, Pampati V, Falco FJ. A randomized, double-blind controlled trial of lumbar interlaminar epidural injections in central spinal stenosis: 2-year follow-up. Pain Physician 2015;18:79-92.

15. Manchikanti L, Cash KA, Pampati V, Falco FJ. Transforaminal epidural injections in chronic lumbar disc herniation: a randomized, double-blind, active-control trial. Pain Physician 2014;17:E489-501.

16. Hawker GA, Mian S, Kendzerska T, French M. Measures of adult pain: Visual Analog Scale for Pain (VAS Pain), Numeric Rating Scale for Pain (NRS Pain), McGill Pain Questionnaire (MPQ), Short-Form McGill Pain Questionnaire (SF-MPQ), Chronic Pain Grade
Scale (CPGS), Short Form-36 Bodily Pain Scale (SF-36 BPS), and Measure of Intermittent and Constant Osteoarthritis Pain (ICOAP). Arthritis Care Res (Hoboken) 2011;63 Suppl 11:S240-52.

17. Chansirinukor W, Maher CG, Latimer J, Hush J. Comparison of the functional rating index and the 18-item Roland-Morris Disability Questionnaire: responsiveness and reliability. Spine (Phila Pa 1976) 2005;30:1415.

18. Grotle M, Brox JI, Vollestad NK. Functional status and disability questionnaires: what do they assess? A systematic review of back-specific outcome questionnaires. Spine (Phila Pa 1976) 2005;30:130-40.

19. Feise RJ, Michael Menke J. Functional rating index: a new valid and reliable instrument to measure the magnitude of clinical change in spinal conditions. Spine (Phila Pa 1976) 2001;26:78-87.

20. Kalff R, Ewald C, Waschke A, Gobisch L, Hopf C. Degenerative lumbar spinal stenosis in older people: current treatment options. Dtsch Arztebl Int 2013;110:613-23.

21. Howe JF, Loeser JD, Calvin WH. Mechanosensitivity of dorsal root ganglia and chronically injured axons: a physiological basis for the radicular pain of nerve root compression. Pain 1977;3:25-41.

22. Cosgrove JL, Bertolet M, Chase SL, Cosgrove GK. Epidural steroid injections in the treatment of lumbar spinal stenosis efficacy and predictability of successful response. Am J Phys Med Rehabil 2011;90:1050-5.

23. Mert T, Gunes Y, Ozcengiz D, Gunay I, Polat S. Comparative effects of lidocaine and tramadol on injured peripheral nerves. Eur J Pharmacol 2006;543:54-62.

24. Persaud N, Strichartz GR. Micromolar lidocaine selectively blocks propagating ectopic impulses at a distance from their site of origin. Pain 2002;99:333-40.

25. Chaplan SR, Bach FW, Shafer SL, Yaksh TL. Prolonged alleviation of tactile allodynia by intravenous lidocaine in neuropathic rats. Anesthesiology 1995;83:77585. 